\title{
Changes in Outpatient Services and Medication Use Following a Non-fatal Opioid Overdose in the West Virginia Medicaid Program
}

\author{
Neel Koyawala, BA, BS ${ }^{1,2}$, Rachel Landis, MPP 3 , Colleen L. Barry, PhD, MPP 2,4,5, \\ Bradley D. Stein, MD, $P h D^{6}$, and Brendan Saloner, $P h D^{2,4,5}$
}

'Johns Hopkins School of Medicine, Baltimore, MD, USA; ${ }^{2}$ Center for Mental Health and Addiction Policy Research, Johns Hopkins Bloomberg School of Public Health, Baltimore, MD, USA; ${ }^{3}$ The George Washington University Trachtenberg School of Public Policy and Public Administration, Washington, DC, USA; ' ${ }^{4}$ epartment of Health Policy and Management, Johns Hopkins Bloomberg School of Public Health, Baltimore, MD, USA; ${ }^{5}$ Department of Mental Health, Johns Hopkins Bloomberg School of Public Health, Baltimore, MD, USA; ${ }^{6}$ RAND Corporation, Pittsburgh, PA, USA.

J Gen Intern Med 34(6):789-91

DOI: $10.1007 / \mathrm{s} 11606-018-4817-8$

(c) Society of General Internal Medicine 2019

\section{INTRODUCTION}

West Virginia leads the USA in opioid overdoses, with a rate more than three times the national average. ${ }^{1}$ Post-overdose medications for opioid use disorder (MOUD) are protective against future deaths, ${ }^{2}$ yet are infrequently prescribed. ${ }^{2-4}$

Mental health disorders increase opioid overdose risk. ${ }^{5}$ Given the substantial burden of psychiatric comorbidities among people who overdose, it is important to understand how both MOUD and treatment for co-occurring mental health conditions change following overdoses.

\section{METHODS}

We obtained West Virginia Medicaid claims data for individuals enrolled under the Affordable Care Act expansion from 2014 to 2016. Opioid overdoses were identified using diagnosis codes for opioid poisoning (965.00-965.02, 965.09, E850.0-E850.2, appropriate T40 codes) as defined by ICD$9 / 10 .{ }^{6}$ Office visits (99x) for opioid use disorder (OUD) (304.00-304.03, 305.50-305.53, F11x) and counseling (H0004, H0031, H0036, H0040, H2036, 90791, 90792, 908x) and diagnoses for mental health conditions were determined using ICD-9/10 and the Current Procedural Terminology codes. Prescription drugs were classified using the First Data Bank three-digit hierarchical ingredient code. The sample was restricted to people continuously Medicaid-enrolled for 6 months prior to and 12 months following an overdose. Paired $t$ tests were used to compare individual receipt of services and medications in months $1-3$ prior to overdose versus other 3-month segments post-overdose. Unadjusted graphical displays were created to provide more detail on month-level trends. Analyses were conducted using STATA (StataCorp), Version 15. This study was exempted by the Johns Hopkins University Institutional Review Board.

Published online January 28, 2019

\section{RESULTS}

Our sample included 301 people enrolled in West Virginia Medicaid who had a non-fatal overdose in 2014-2015. The sample was $60 \%$ male and $91 \%$ non-Hispanic White with mean age of 34.5 years; $54 \%$ of people had a diagnosis of depression, anxiety disorder, bipolar disorder, or schizophrenia (not shown).

By 12 months post-overdose, individuals were more likely to receive buprenorphine $(4.7 \%$ versus $8.3 \%$, $P=.02)$ and less likely to receive opioid analgesics (35.9\% versus $23.6 \%, P<.001)$, or benzodiazepines ( $15.9 \%$ versus $11.6 \%, P=.03$ ), compared to the 3 months prior to overdose. They were also less likely to receive mental health counseling $(23.3 \%$ versus $15.3 \%, P=<.01)$. Differences were not significant for OUD office visits and receipt of naltrexone, antidepressants, antipsychotics, antianxiety, or antimania drugs (Table 1).

As month-level unadjusted analysis shows, OUD office visits spiked in the month of overdose, but then resumed pre-overdose trends (Fig. 1). In the 6 months after a nonfatal overdose, the proportion of people receiving buprenorphine increases by approximately 0.5 percentage points per month, while the proportion of people receiving opioid analgesics decreases by approximately 1.2 percentage points per month. At 12 months post-overdose, 7.3\% of people received buprenorphine and $15.3 \%$ received opioid analgesics.

\section{DISCUSSION}

Focusing on the state with the highest overdose rate, we find that use of buprenorphine significantly increased after a nonfatal overdose, but remained very low. Less than $10 \%$ of people every month post-overdose received MOUD or mental health counseling, which is below rates reported in other states. ${ }^{2,3} \mathrm{In}$ our analysis, MOUD only includes buprenorphine and naltrexone because West Virginia Medicaid did not cover methadone. Despite high rates of mental health comorbidities, people saw 
Table 1 Services and Medications Pre- and Post-opioid Overdose by Time Segment

\begin{tabular}{|c|c|c|c|c|c|c|c|c|c|c|c|}
\hline & $\begin{array}{c}\text { MONTHS 1-3 } \\
\text { PRE- } \\
\text { OVERDOSE }\end{array}$ & $\begin{array}{r}\text { MONTHS } \\
\text { OVERL }\end{array}$ & $\begin{array}{l}\text { 4-6 PRE- } \\
\text { OSE }\end{array}$ & $\begin{array}{r}\text { MONTHS } 1 \\
\text { OVER }\end{array}$ & $\begin{array}{l}\text { 1-3 POST- } \\
\text { DOSE }\end{array}$ & $\begin{array}{r}\text { MONTHS } \\
\text { OVER }\end{array}$ & $\begin{array}{l}\text { 4-6 POST- } \\
\text { DOSE }\end{array}$ & $\begin{array}{r}\text { MONTHS } \\
\text { OVER }\end{array}$ & $\begin{array}{l}\text { 7-9 POST- } \\
\text { RDOSE }\end{array}$ & $\begin{array}{r}\text { MONTHS } 10 \\
\text { OVER }\end{array}$ & $\begin{array}{l}\text { 0-12 POST- } \\
\text { DOSE }\end{array}$ \\
\hline & No. (\%) & No. (\%) & P Value & No. (\%) & P Value & No. (\%) & P Value & No. (\%) & P Value & No. (\%) & P Value \\
\hline Counseling and office visits for 0 & & & & & & & & & & & \\
\hline Office visit related to OUD & $44(14.6)$ & $34(11.3)$ & .07 & $39(13)$ & .46 & $40(13.3)$ & .60 & $38(12.6)$ & .41 & $38(12.6)$ & .38 \\
\hline Mental health counseling & $70(23.3)$ & $61(20.3)$ & .26 & $57(18.9)$ & .11 & $48(15.9)$ & $0.01 *$ & $49(16.3)$ & $0.02 *$ & $46(15.3)$ & $.005^{* *}$ \\
\hline Medications for OUD & & & & & & & & & & & \\
\hline Receipt of buprenorphine & $14(4.7)$ & $12(4.0)$ & .53 & $13(4.3)$ & .82 & $20(6.6)$ & .18 & $26(8.6)$ & $.01 *$ & $25(8.3)$ & $.02 *$ \\
\hline Receipt of naltrexone & $8(2.7)$ & $2(0.7)$ & $.03 *$ & $5(1.7)$ & .37 & $4(1.3)$ & .21 & $5(1.7)$ & .26 & $6(2.0)$ & .53 \\
\hline $\begin{array}{l}\text { Buprenorphine or naltrexone } \\
\text { (MOUD) }\end{array}$ & $22(7.4)$ & $14(4.7)$ & $.05^{*}$ & $17(5.6)$ & .35 & $24(8.0)$ & .72 & $30(10.0)$ & .14 & $31(10.3)$ & .09 \\
\hline Opioids and benzodiazepines & & & & & & & & & & & \\
\hline Receipt of opioid analgesic & 108 (35.9) & $118(39.2)$ & .26 & 102 (33.9) & .51 & 66 (21.9) & $<.001 * * *$ & $75(24.9)$ & $<.001 * * *$ & $71(23.6)$ & $<.001 * * *$ \\
\hline Receipt of benzodiazepine & $48(15.9)$ & $46(15.3)$ & .66 & $38(12.6)$ & .06 & $39(13)$ & .12 & $40(13.3)$ & .21 & $35(11.6)$ & $.03^{*}$ \\
\hline Benzo+opioid in same month & $30(10)$ & $34(11.3)$ & .35 & $20(6.6)$ & $0.03 *$ & $20(6.6)$ & $.04 *$ & $21(7)$ & .11 & $17(5.6)$ & $.01^{*}$ \\
\hline Other psychiatric drugs & & & & & & & & & & & \\
\hline Receipt of antidepressant & $110(36.5)$ & $98(32.6)$ & .08 & 102 (33.9) & .29 & $98(32.6)$ & .12 & 95 (31.6) & .05 & $95(31.6)$ & .05 \\
\hline Receipt of antipsychotic & $39(13.0)$ & $33(11.0)$ & .18 & $43(14.3)$ & .43 & $43(14.3)$ & .52 & $41(13.6)$ & .75 & $42(14.0)$ & .65 \\
\hline Receipt of antianxiety drug & $56(18.6)$ & $52(17.3)$ & .43 & $47(15.6)$ & .12 & $46(15.3)$ & .12 & $48(15.9)$ & .24 & $46(15.3)$ & .16 \\
\hline Receipt of antimania drug & $5(1.7)$ & $5(1.7)$ & 1.00 & $7(2.3)$ & .42 & $6(2.0)$ & .66 & $4(1.3)$ & .56 & $7(2.3)$ & .42 \\
\hline
\end{tabular}

$N=301$ non-fatal opioid overdose patients. Analysis uses West Virginia Medicaid claims data for individuals enrolled under the Affordable Care Act expansion from 2014 to 2016. Month 0 represents month of overdose. Percentages represent percent of individuals with receipt of any drug/service in time segment. Paired t tests compare individual receipt of services and medications in months 1-3 prior to overdose versus other 3-month segments post-overdose. Overdose poisoning codes $=965.00-965.02,965.09$, E850.0-E850.2, T40.yX1A, T40.yX1D, T40.yX1S, T40.yX4A, T40.yX4D, T40.yX4S, T40.yS2A, T40.yX2A, T40.601A, T40.601D, T40.601S, T40.604A, T40.604D, T40.604S, T40.691A, T40.691D, T40.691S, T40.694A, T40.694D,

T40.694S; $y=0,1,2,3,4,6$. Opioid use disorder (OUD) codes $=304.00-304.03,305.50-305.53$, F11x. Office visit codes $=99 x$. Mental health counseling codes = H0004, H0036, H0031, H0040, H2036, 90791-2, 908x. Buprenorphine HIC= H3W. Naltrexone HIC = H3T. Opioid analgesic HIC $=H 3 x$ except H3C, H3D, H3E, H3F, H3I, H3K, H3L, H3O, H3T, H3V, H3W, H3Y. Benzodiazepine HIC = H2F and "alprazozolam," "lorazepam," "clonazepam," "diazepam," "bromazepam." Antidepressant HIC = H2S, H2U, H2W, H7B, H7C, H7D, H8P, H8T. Antipsychotic HIC = H2G, H7O, $H 7 P, H 7 R, H 7 S, H 7 T, H 7 U, H 7 X, H 8 W$. Antianxiety HIC $=H 2 F$. Antimania HIC = H2M. Drug claims without HIC codes were labeled using list of drug names generated from HIC algorithm for that drug. Proportions were compared using paired t tests. P value $<.05^{*},<.01 * *,<.001 * *$

little to no change in receipt of counseling or medications for OUD or mental health conditions post-overdose.

With less than $10 \%$ of people receiving MOUD postoverdose among West Virginia Medicaid enrollees, a nonfatal overdose presents a major, under-utilized opportunity to increase treatment for opioid use disorder. Given the high burden of psychiatric comorbidities and the role of counseling in medication-assisted treatment for opioid use disorder, low rates of and declines in mental health counseling postoverdose also presents an important opportunity to improve treatment for people who experience an opioid overdose. Our study is the first to investigate changes in treatment for psychiatric comorbidities following a non-fatal overdose. Limitations of the study include restriction to those meeting continuous Medicaid eligibility requirements and inability of claims data to capture care not billed. Efforts to reduce risk of 
a Counseling and office visits for opioid use disorder

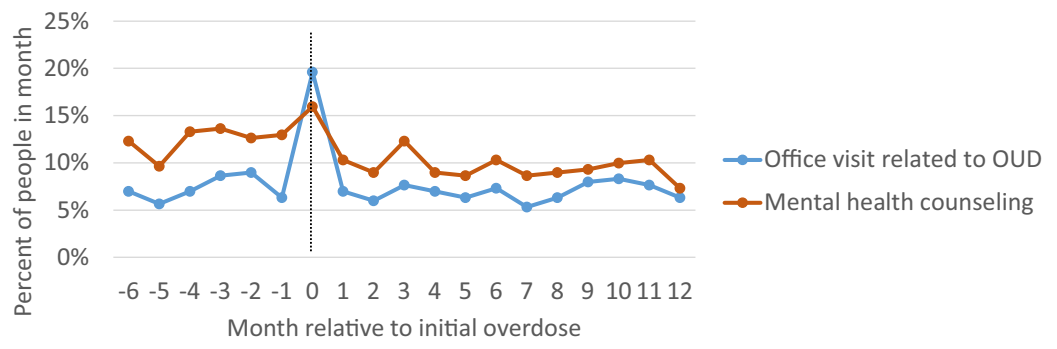

b Opioids and medications for opioid use disorder

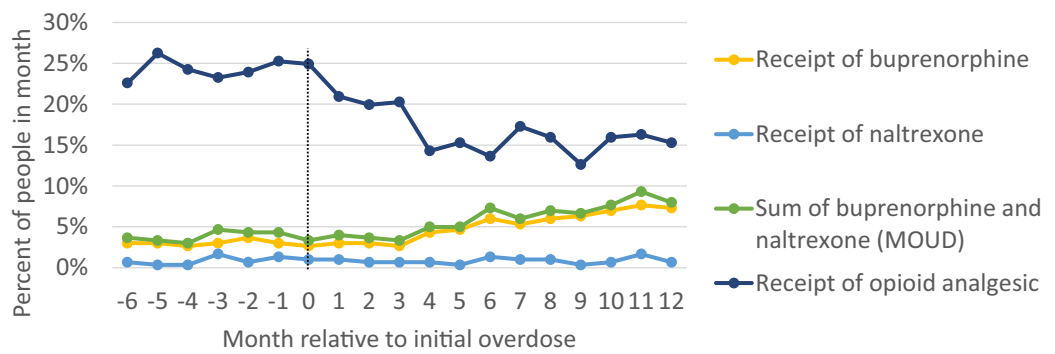

Notes | N=301 non-fatal opioid overdose patients. Analysis uses West Virginia Medicaid claims data for individuals enrolled under the Affordable Care Act expansion from 2014 to 2016. Month 0 represents month of overdose. Percentages represent percent of individuals with receipt of any drug/service in month

Figure 1 Monthly receipt of services and medications pre- and post-opioid overdose.

complications post-overdose should strive for adequate use of both MOUD and treatments for psychiatric conditions that are highly comorbid with OUD.

Acknowledgements: Funding disclosure: Dr. Saloner receives funding from the National Institute on Drug Abuse (KO1 DA042139) and Dr. Stein receives funding from the National Institute on Drug Abuse (P50DA046351, R01DA045800-01).

Corresponding Author: Neel Koyawala, BA, BS; Center for Mental Health and Addiction Policy ResearchJohns Hopkins Bloomberg School of Public Health, Baltimore, MD, USA (e-mail: nkoyawala@jhmi.edu).

\section{Compliance with Ethical Standards:}

Conflict of Interest: The authors declare that they do not have a conflict of interest.

Publisher's Note: Springer Nature remains neutral with regard to jurisdictional claims in published maps and institutional affiliations.

\section{REFERENCES}

1. National Institute on Drug Abuse. West Virginia Opioid Summary: OpioidRelated Overdose Deaths.; 2018. https://www.drugabuse.gov/drugsabuse/opioids/opioid-summaries-by-state/west-virginia-opioid-summary. Accessed 6 November 2018.

2. Larochelle MR, Bernson D, Land T, et al. Medication for opioid use disorder after nonfatal opioid overdose and association with mortality: a cohort study. Ann Inter Med. 2018.

3. Frazier W, Cochran G, Lo-Ciganic W-H, et al. Medication-assisted treatment and opioid use before and after overdose in pennsylvania medicaid. JAMA. 2017;318(8):750-752.

4. Larochelle MR, Liebschutz JM, Zhang F, Ross-Degnan D, Wharam JF Opioid prescribing after nonfatal overdose and association with repeated overdose: a cohort study. Ann Intern Med. 2016;164(1): 1.

5. Bohnert ASB, Ilgen MA, Ignacio RV, McCarthy JF, Valenstein M, Blow FC. Risk of death from accidental overdose associated with psychiatric and substance use disorders. Am J Psychiatry. 2012;169(1):64-70.

6. Moore B, Barrett M. Case Study: Exploring How Opioid-Related Diagnosis Codes Translate From ICD-9-CM to ICD-10-CM. U.S. Agency for Healthcare Research and Quality; 2017. https://www.hcup-us.ahrq.gov/ reports/methods/methods.jsp. Accessed 6 November 2018. 